\title{
BIOEROSIÓN EN ERIZOS IRREGULARES (CLYPEASTEROIDEA) DEL MIOCENO SUPERIOR EN EL SECTOR SUROCCIDENTAL DE LA CUENCA DEL GUADALQUIVIR (PROVINCIA DE SEVILLA)
}

\author{
Ana SANTOS ${ }^{1}$, Eduardo MAYORAL $L^{2}$, Fernando \\ MUÑIZ3 , Ildefonso BAJO y Olga ADRIAENSENS ${ }^{5}$ \\ ${ }^{1}$ Centro de Investigação Marinha e Ambiental, Universidade do Algarve, \\ Faculdade de Ciências do Mar e do Ambiente, Campus de Gambelas, 8000- \\ 117 Faro, Portugal. aasantos@ualg.pt \\ ${ }^{2}$ Departamento de Geodinámica y Paleontología, Facultad de Ciencias Expe- \\ rimentales, Universidad de Huelva, Campus del Carmen, Avda. de las Fuer- \\ zas Armadas s/n 21071 Huelva, España.mayoral@uhu.es \\ ${ }^{3}$ Grupo de Investigación RNM 316 "Tectónica y Paleontología”, Universi- \\ dad de Huelva, España. gyrolithes@yahoo.es \\ ${ }^{4}$ Fuerteventura, 2, 41700 Dos Hermanas, Sevilla, España. ildebajo@wanadoo.es \\ ${ }^{5}$ Virgen de la Antigua, 10, 41011 Sevilla, España.
}

Santos, A., Mayoral, E., Muñiz, F., Bajo, I. y Adriaensens, O. 2003. Bioerosión en erizos irregulares (Clypeasteroidea) del Mioceno Superior en el sector suroccidental de la cuenca del Guadalquivir (Provincia de Sevilla). [Bioerosion on irregular sea urchins (Clypeasteroidea) from the Upper Miocene in the southwestern sector of the Guadalquivir Basin (province of Sevilla).] Revista Española de Paleontología, 18 (2), 131-141. ISSN 0213-6937.

\begin{abstract}
Bioerosive structures recorded on several species of echinoids of the Order Clypeasteroidea have been studied. These species are Clypeaster cermenatii Checchia-Rispoli, 1925, C. marginatus Lamarck, 1816, C. portentosus Desmoulins, 1837, C. brevior Seguenza, 1879, C. aichinoi Checchia-Rispoli, 1925, C. tauricus Desor, 1858, C. franchii Checchia-Rispoli, 1925, C. altus Klein, 1734 and Clypeaster sp., all of them from the northern border of the Guadalquivir Basin between Peñaflor and Alcolea del Río (Sevilla province), belonging to Upper Miocene (Late Tortonian) in age. Evidences of predation behavior showing search for food (borings, breakings and/or test deepening), and domicile are analyzed. The ichnotaxa Oichnus simplex Bromley, 1981 and Oichnus ichnosp. indet. and significant test breakings produced by sharp incisions or bitting predation are included inside the first category. Usually, reparation evidences are observed, consisting in cementation of calcareous fragments and secretions of secondary test material resulting in spectacular teratologic growths. These records point to predators related probably to cassid gastropods, as several crustaceans, echinoderms and fishes. In the second category are included the icnotaxa Gastrochaenolites cf. digujus Kelley and Bromley, 1984 and Maeandropolydora sulcans Voigt, 1965, representing the activity of lithophagous bivalves and polychaeta annelids, respectively.
\end{abstract}

Keywords: Clypeasteroid echinoidea, bioerosion, Upper Miocene, Guadalquivir basin, Sevilla, Spain.

\section{RESUMEN}

Se han estudiado las evidencias de bioerosión registradas en diferentes equinoideos pertenecientes al orden Clypeasteroidea, concretamente en las especies Clypeaster cermenatii Checchia-Rispoli, 1925, C. marginatus Lamarck, 1816, C. portentosus Desmoulins, 1837, C. brevior Seguenza, 1879, C. aichinoi Checchia-Rispoli, 1925, C. tauricus Desor, 1858, C. franchii Checchia-Rispoli, 1925, C. altus Klein, 1734 y Clypeaster sp., procedentes del Mioceno Superior (Tortoniense superior) del borde septentrional de la Cuenca del Guadalquivir, en el sector comprendido entre Peñaflor y Alcolea del Río, en la provincia de Sevilla. Se han analizado las evidencias que implican comportamientos de búsqueda de alimento por depredación (perforaciones, roturas y/o hundimientos en el caparazón), y de domicilio o refugio. Dentro de la primera categoría se han identificado los 
icnotaxones Oichnus simplex Bromley, 1981 y Oichnus ichnosp. indet., así como roturas importantes del caparazón relacionadas con profundas incisiones o mordiscos por parte de depredadores. Asociado a estas roturas se encuentran evidencias de reparación por cementación de fragmentos calcáreos y zonas de secreción anormal de material secundario que han dado lugar a crecimientos teratológicos muy espectaculares. Estos registros ponen de manifiesto la existencia de depredadores relacionados muy probablemente con gasterópodos cassididos, así como con diversos crustáceos, equinodermos y peces. En la segunda categoría, se han identificado los icnotaxones Gastrochaenolites cf. digujus Kelley y Bromley, 1984 y Maeandropolydora sulcans Voigt, 1965, relacionados con la actividad de bivalvos litófagos y anélidos poliquetos respectivamente.

Palabras clave: Equinoideos clypeasteroideos, Bioerosión, Mioceno Superior, Cuenca del Guadalquivir, Sevilla, España.

\section{INTRODUCCIÓN}

Los equinoideos irregulares, especialmente los que pertenecen al orden Clypeasteroidea, son, por la propia morfología de sus caparazones y por su particular forma de vida, un grupo de organismos que se constituyen en importantes sustratos, suficientemente rígidos y consistentes (sobre todo una vez muertos), como para soportar la instalación de numerosos organismos de hábitos incrustantes (crustáceos balanomorfos, algas coralinas, briozoos, anélidos, bivalvos ostréidos, etc) y perforantes (esponjas cliónidas, anélidos, bivalvos litófagos, gasterópodos, etc). Estos caparazones no sólo son objeto de colonizaciones pasivas post-mortem, sino que también pueden verse afectados a lo largo de su vida por organismos parásitos o, más frecuentemente, por depredadores.

En todos los casos, el registro de estas interacciones suele ser muy numeroso, lo que permite establecer reconstrucciones paleoecológicas muy precisas, tanto a nivel de relaciones individuales como las que puedan producirse entre éstos y el medio.

En el presente trabajo se abordan las principales estructuras bioerosivas registradas sobre diferentes especies de Clypeaster Lamarck, 1801, halladas y descritas en el área de estudio por Bajo (2002), y que responden a un comportamiento de búsqueda de refugio o domicilio, así como a conductas que tienen que ver con la depredación y/o el parasitismo.

\section{SITUACIÓN GEOGRÁFICA Y GEOLÓGICA}

El material estudiado procede de las localidades de Burguillos, Villanueva del Río y Minas, Alcolea del Río, Lora del Río y Peñaflor, todas ellas situadas en la provincia de Sevilla, al N-NE de la capital y ubicadas en la margen derecha del Guadalquivir (Fig. 1). La mayor parte del material procede del área de Villanueva del Río y Minas, extraído de varias canteras que se sitúan, unas a unos $3 \mathrm{~km}$ al NE del núcleo urbano y otras en la rivera del Huéznar, a $1 \mathrm{~km}$ al $\mathrm{E}$ de la población. Las áreas de Alcolea y Lora del Río también han suministrado gran cantidad de material. En la primera, en una serie de canteras situadas a unos $2 \mathrm{~km}$ al $\mathrm{N}$ de la población, y en la

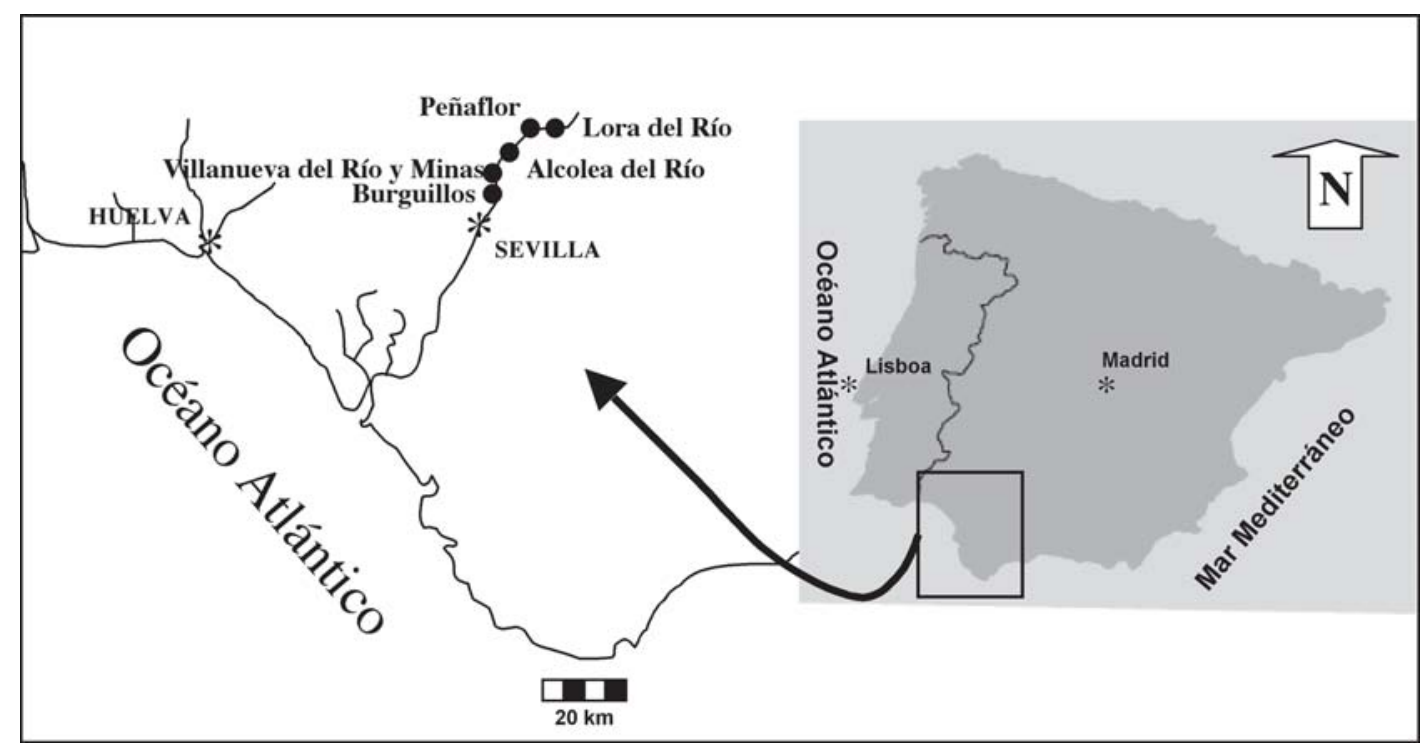

Figura 1. Localización geográfica de los yacimientos. Geographical location of the sites. 
segunda, en una cantera abandonada a unos $3 \mathrm{~km}$ al $\mathrm{N}$ de Lora, así como en el corte de la carretera SE-147 que une Lora con La Puebla de los Infantes.

Desde el punto de vista geológico, estas áreas se engloban dentro de una facies bioclástica típica denominada localmente como Unidad Terrígena Basal, cuya edad es Mioceno Superior (Tortoniense superior; Sierro et al., 1990), y que se extiende, con ligeras variaciones composicionales y de toponimias, a lo largo de todo el borde septentrional de la Cuenca del Guadalquivir. Las especies de Clypeaster estudiadas proceden de esta Unidad, si bien algunas se han localizado también en niveles estratigráficos superiores, pertenecientes a otras unidades.

\section{MATERIAL ESTUDIADO}

En toda el área de estudio se han recogido un total de 72 erizos, de los que sólo han sido objeto de análisis aquellas especies que presentaban alguna evidencia de perforación o rotura. Entre los ejemplares que presentan bioerosión, se encuentran: Clypeaster portentosus Desmoulins, 1837, con veintiún individuos, C. marginatus Lamarck, 1816, con cuatro individuos, C. cermenatii Checchia-Rispoli, 1925, con dieciséis individuos, y Clypeaster sp., con catorce individuos. Entre los restantes se han obtenido: tres ejemplares de C. brevior Seguenza, 1879, con sólo un individuo perforado, cuatro de C. altus Klein, 1734, también con sólo un individuo perforado, y sin ningún tipo de evidencias, tres ejemplares de C. franchii Checchia-Rispoli, 1925, un ejemplar de C. aichinoi Checchia-Rispoli, 1925, y uno de $C$. tauricus Desor, 1858.

Todos los ejemplares se hallan depositados en el Museo de Geología de la Universidad de Sevilla con las siglas MGUS2001 a MGUS-2072.

\section{ESTRATIGRAFÍA}

La Unidad Terrígena Basal varía de potencia en función de la posición que ocupe respecto al borde de la cuenca y de la litología y/o granulometría de las facies, si bien todas ellas responden a una constitución básicamente bioclástica. Se expondrá una sucesión de síntesis basada en la sección de Villanueva del Río y Minas, ya que ésta ocupa una posición intermedia dentro del área de estudio y es una de las que ha suministrado más y mejor material, siendo por ello muy representativa. La serie consta desde el muro hasta el techo de los siguientes intervalos (Fig. 2):

Intervalo 1. Desde 6-7 $\mathrm{m}$ de potencia en el yacimiento de Burguillos hasta $54 \mathrm{~m}$ en Alcolea del Río. Son arenas medias muy bioturbadas con intercalaciones de arenas gruesas a muy gruesas, gravas y microconglomerados de espesor decimétrico, formados por cantos de pizarras y cuarcitas, con niveles de ostréidos, pectínidos, cirrípedos y acumulaciones de Heterostegina sp. hacia la parte media-alta del intervalo. Los niveles más cementados y de mayor ta-

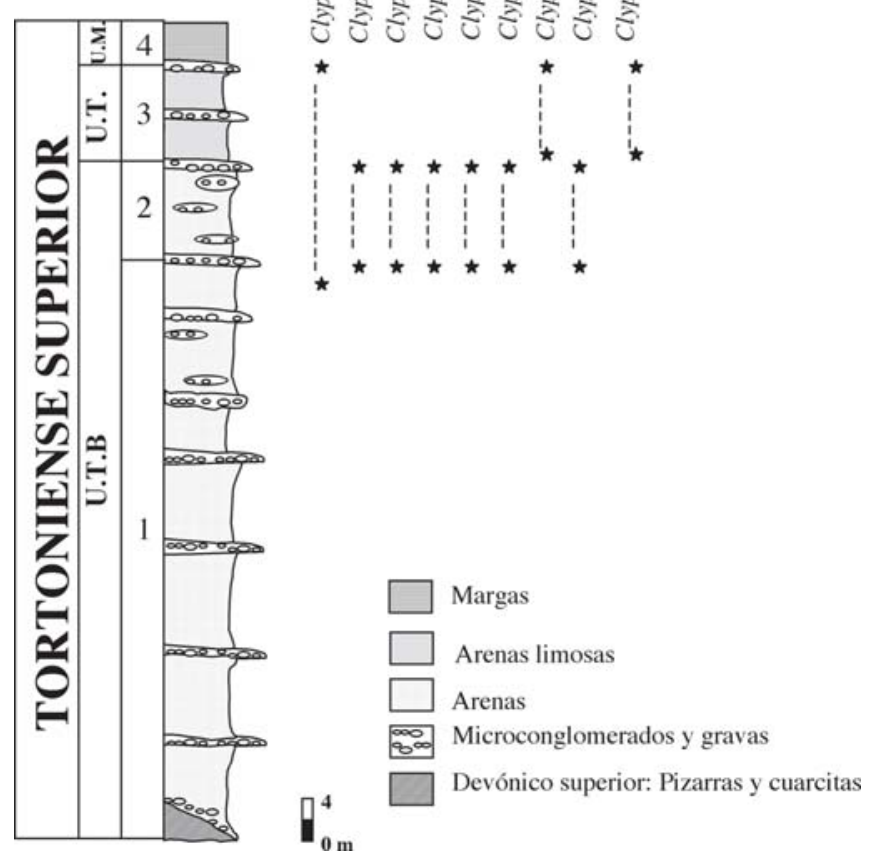

Figura 2. Columna litoestratigráfica sintética y distribución vertical de las especies de Clypeaster. U.T.B.: Unidad Terrígena Basal. U.T.: Unidad de Transición. U.M.: Unidad Margosa.

Synthetic lithostratigraphical section and vertical distribution of the Clypeaster species. U.T.B.: Basal Terrigenous Unit. U.T.: Transition Unit. U.M.: Marly Unit.

maño de grano presentan frecuentemente estructuras de bioturbación representadas por Ophiomorpha nodosa Lundgren, 1891. Hacia techo pasan a ser arenas medias con megaestratificación cruzada de base curva, las cuales presentan esporádicamente dientes de seláceos (Isurus hastalis [Agassiz, 1843]), y de perciformes (Sparus sp.), junto con abundantes restos de cetáceos y moldes de bivalvos (Pholadomya alpina Matheron, 1842), así como restos leñosos con Teredolites ichnosp. indet. En el techo de este tramo aparecen los primeros registros de Clypeaster portentosus.

Intervalo $2.10 \mathrm{~m}$ de arenas muy gruesas, microconglomerados y gravas, con grandes Ophiomorpha. Hacia techo aparecen canales de base erosiva con arenas gruesas que presentan estratificación cruzada y que presentan restos de cetáceos y de gasterópodos (Scaphander lignaria (Linné, 1758), Ficus sp., Conus sp.), bivalvos (Gigantopecten albinus von Teppner, 1915, Anomia ephippium Linné, 1758, y Leporimetis lacunosa Chemnitz, 1792), junto con la mayor parte de los erizos estudiados, estando representados Clypeaster brevior, $C$. cermenatii, $C$. aichinoi, $C$. tauricus, $C$. franchii y $C$. altus. Todos ellos son ejemplares de gran tamaño, con formas elevadas y bordes gruesos, normalmente en posición de máximo equi- 
librio mecánico (con la cara aboral dispuesta hacia arriba y la oral paralela al plano de estratificación).

Intervalo 3. Unidad de Transición, constituida por $10 \mathrm{~m}$ de arenas limosas finas, intensamente bioturbadas con intercalaciones de arenas gruesas muy ricas en Heterostegina sp., en las que aparecen bivalvos articulados (Flabellipecten solarium (Lamarck, 1814), Chlamys multistriata (Poli, 1795), Flabellipecten flaterculus (Sowerby, 1841), Flabellipecten sp.) y abundantes equinodermos, especialmente en la base y techo de este intervalo, donde se han recogido Clypeaster marginatus, Clypeaster sp. en la base, y en el techo, junto a ellos, C. portentosus. Suelen aparecer normalmente en posición de máximo equilibrio mecánico, junto con restos de vertebrados marinos. Hacia techo las arenas pasan a ser glauconíticas, presentando localmente niveles de arenas de grano medio. Este intervalo aflora tan sólo en las localidades de Burguillos y Villanueva del Río y Minas.

Intervalo 4. Unidad Margosa, constituida por $4 \mathrm{~m}$ visibles de margas grises, que en la base son limos arenosos muy ricos en glauconita y en restos de cetáceos y dientes de seláceos.

\section{PALEOICNOLOGÍA SISTEMÁTICA}

\section{Oichnus simplex Bromley, 1981}

Figs. 3 a, b; 7 a, b.

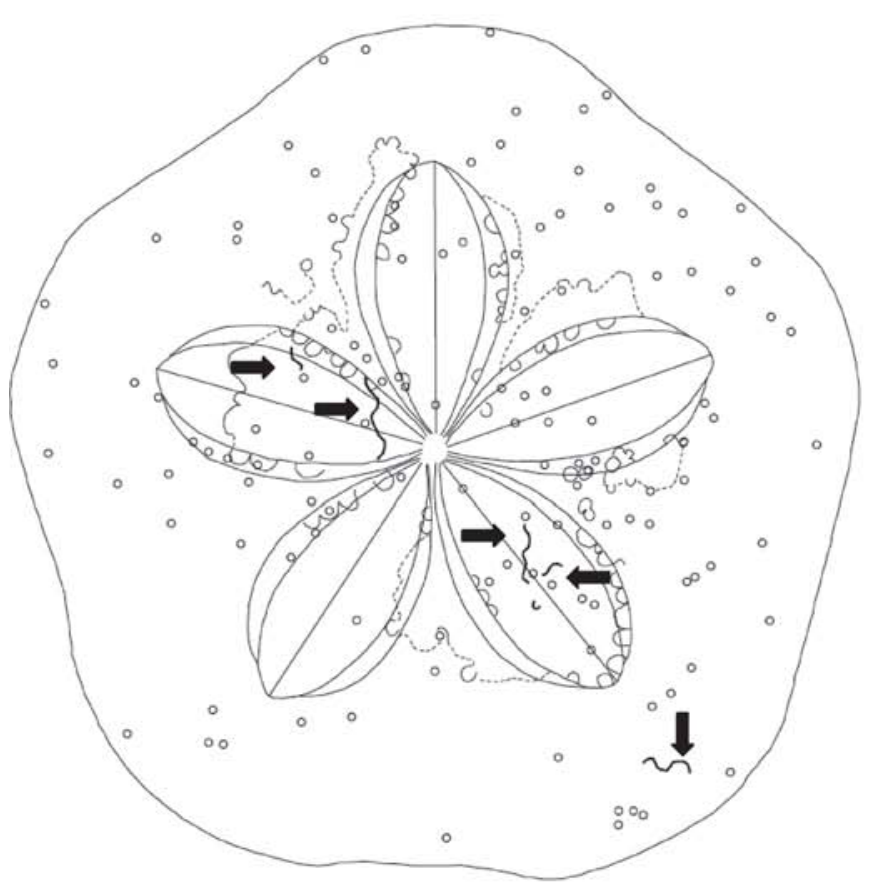

(a)
Descripción: Orificios cilíndricos simples o subcilíndricos, cuyo eje es más o menos perpendicular a la superficie del sustrato, de contorno redondeado, muy regulares, cuyo diámetro varía entre $3 \mathrm{~mm}$ y $5 \mathrm{~mm}$, con una media en torno a 3,8-4 mm.

Observaciones: Se han registrado un total de 202 evidencias, de las cuales ciento noventa y siete se sitúan en la cara aboral y tan sólo cinco en la cara oral, siendo el sustrato más afectado Clypeaster sp. (Tabla 1). Pueden aparecer en cualquier parte de la cara aboral, aunque suelen ser más frecuentes sobre los ambulacros petaloideos, especialmente en sus zonas poríferas, donde además suelen presentar sus diámetros máximos. En estos casos casi siempre la densidad de perforación en el área afectada es muy grande, lo que hace que se solapen unas perforaciones con otras, destruyendo literalmente la zona porífera de los ambulacros (Figs. 3a, 7 a, b).

\section{Oichnus ichnosp. indet.}

Fig. 4

Descripción: Orificios subcilíndricos de contorno subcircular o alargado, a modo de depresiones, irregulares, con un diámetro mínimo medio de aproximadamente $1 \mathrm{~mm}$ y uno máximo medio de $3,5 \mathrm{~mm}$.

Observaciones: Se han registrado un total de 51 evidencias, siempre en la cara aboral, siendo C. cermenatii el sustrato

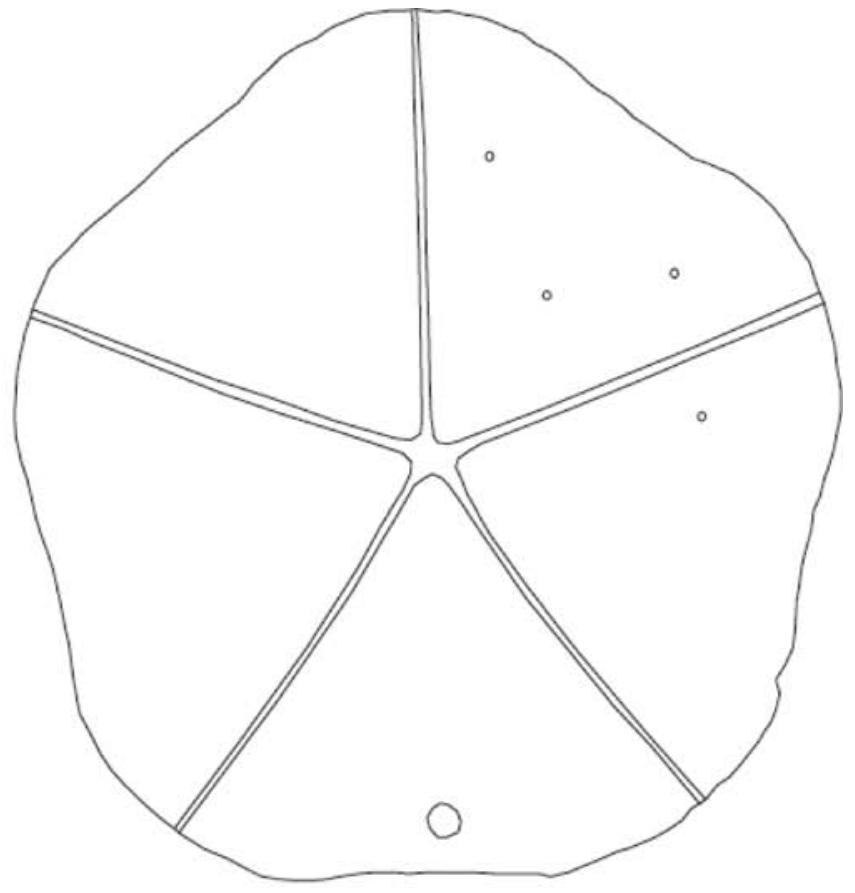

(b)

Figura 3. Diagramas de contorno para Clypeaster que muestran la distribución de Oichnus simplex Bromley, 1981 (círculos blancos) y Maeandropolydora sulcans Voigt, 1965 (flechas). a: Superficie aboral. b: Superficie oral.

Sketches of the Clypeaster test showing the distribution of Oichnus simplex Bromley, 1981 (white circles) and Maeandropolydora sulcans Voigt, 1965 (arrows). a: Aboral surface. b: Oral surface. 


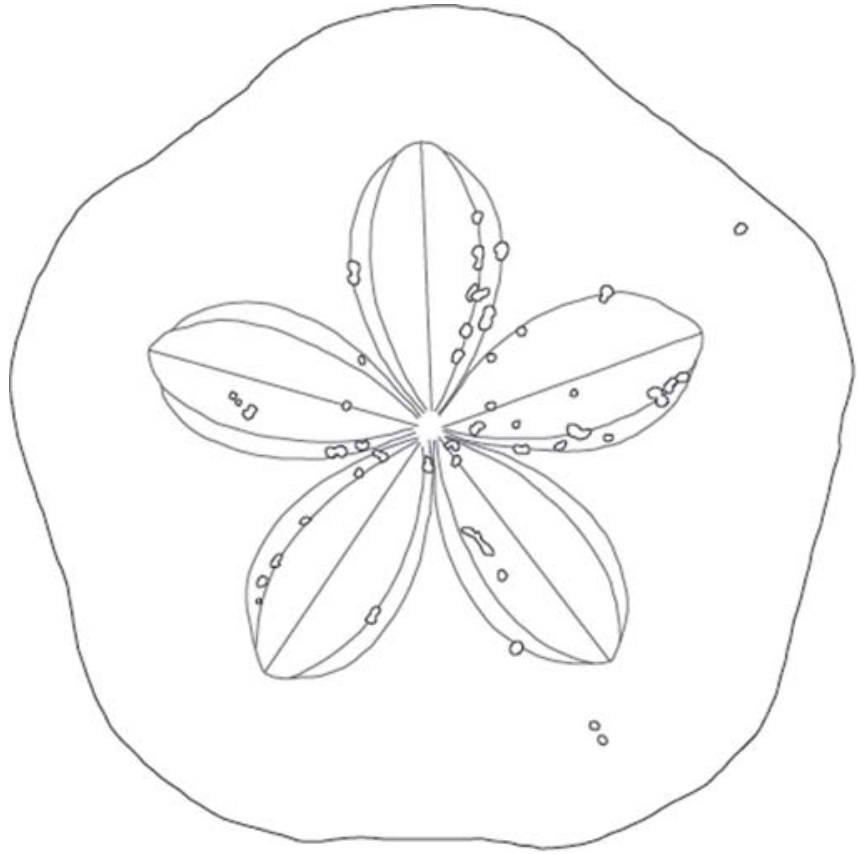

Figura 4. Diagrama de contorno con la distribución de Oichnus ichnosp. indet (círculos blancos). Superficie aboral. Sketch showing the distribution of Oichnus ichnosp. indet (white circles). Aboral surface.

más afectado (Tabla 1, Fig. 4). Aparecen con una disposición similar a $O$. simplex, casi siempre en la zona petaloidea de los ambulacros. La irregularidad de estas perforaciones está en muchos casos relacionada con estados de conservación deficientes, mostrando inicios de fenómenos de disolución. En ningún caso se han observado características que puedan relacionarlos con otras icnoespecies comunes de Oichnus, como O. paraboloides Bromley, 1981 u O. ovalis Bromley, 1993, siendo más probable que fueran inicialmen- te $O$. simplex, cuyo estado de conservación actual impide una determinación icnotaxonómica precisa.

\section{Gastrochaenolites cf. digujus \\ Kelley y Bromley, 1984 \\ Figs. 5 a, b; 7c, f}

\begin{abstract}
Descripción: Perforaciones en forma de saco, que presentan al inicio una trayectoria verticalizada durante los dos o tres primeros milímetros de profundización en el sustrato, por lo que muestran, cuando no están erosionadas, la típica abertura circular o en forma de ocho, con un diámetro que varía entre 1-2 mm como mínimo, a 8-9 mm como máximo. Conecta hacia el interior mediante un cuello estrecho, que da paso a una cámara principal elongada u ovoidal que se dispone paralela al sustrato, aprovechando al máximo el espesor de éste, por lo que la madriguera adopta una posición subparalela o inclinada en función de cómo lo haga el caparazón (Figs. 7e, f). La longitud de la cavidad principal varía entre 5 y $21 \mathrm{~mm}$ y su sección transversal es circular. Aparecen de forma mayoritaria en la zona petaloidea de la cara aboral.
\end{abstract}

Observaciones: Estos registros constituyen las estructuras bioerosivas más abundantes (261 registros), siendo $C$. cermenatii el equinoideo que presenta el mayor número de ellas (163), apareciendo mayoritariamente en la cara aboral (Tabla 1) y casi siempre restringidas a la zona petaloidea de los ambulacros o en sus inmediaciones. (Figs. 5a, 7c). Los ejemplares presentan características muy próximas a la icnoespecie $G$. digujus, en especial en lo referente a la región de la abertura, cuello y sección transversal. En la mayoría de los casos, el grado de conservación impide hacer observaciones más precisas que permitan establecer con total fiabilidad la asignación sistemática a la mencionada icnoespecie. Respecto a las otras icnoespecies de Gastrochaenolites, las diferencias son muy

\begin{tabular}{|c|c|c|c|c|c|c|c|c|}
\hline Especies & $\begin{array}{c}N^{0} \text { total } \\
\text { ejemplares }\end{array}$ & $\begin{array}{l}\text { Oichnus } \\
\text { simplex }\end{array}$ & \begin{tabular}{|c|} 
Oichnus \\
ichnosp. \\
indet.
\end{tabular} & Mordiscos & Impactos & $\begin{array}{c}\text { Gastrochaenolites } \\
\text { cf. digujus }\end{array}$ & $\begin{array}{c}\text { Maeandropolydora } \\
\text { sulcans }\end{array}$ & \\
\hline \multirow{2}{*}{ C. cermenatii } & \multirow{2}{*}{16} & $22(2)$ & $38(1)$ & $3(1)$ & & $163(12)$ & $6(2)$ & Aboral \\
\hline & & & & & & $1(1)$ & & Oral \\
\hline \multirow{2}{*}{ C. marginatus } & \multirow{2}{*}{4} & $33(1)$ & & & & $4(3)$ & & Aboral \\
\hline & & & & & & & & Oral \\
\hline \multirow{2}{*}{ C. portentosus } & \multirow{2}{*}{21} & $19(1)$ & & & & $50(19)$ & & Aboral \\
\hline & & & & & $4(1)$ & & & Oral \\
\hline \multirow{2}{*}{ C. altus } & \multirow{2}{*}{1} & & & & & $2(1)$ & & Aboral \\
\hline & & & & & & & & Oral \\
\hline \multirow{2}{*}{ C. brevior } & \multirow{2}{*}{1} & & & & & $2(1)$ & & Aboral \\
\hline & & & & & & & & Oral \\
\hline \multirow{2}{*}{ Clypeaster sp. } & \multirow{2}{*}{14} & $123(3)$ & $13(2)$ & $5(3)$ & & $35(5)$ & & Aboral \\
\hline & & $5(1)$ & & & $2(1)$ & $4(1)$ & & Oral \\
\hline Total & 57 & $202(38 \%)$ & $51(9.5 \%)$ & $8(1.5 \%)$ & $6(1 \%)$ & $261(49 \%)$ & $6 \quad(1 \%)$ & \\
\hline
\end{tabular}

Tabla 1. Cuantificación de las estructuras bioerosivas encontradas en Clypeaster. Entre paréntesis se indica el número de ejemplares que presentan la evidencia correspondiente.

Quantification of the bioerosive structures found on Clypeaster. Parentheses point to the number of specimens with the corresponding evidence. 

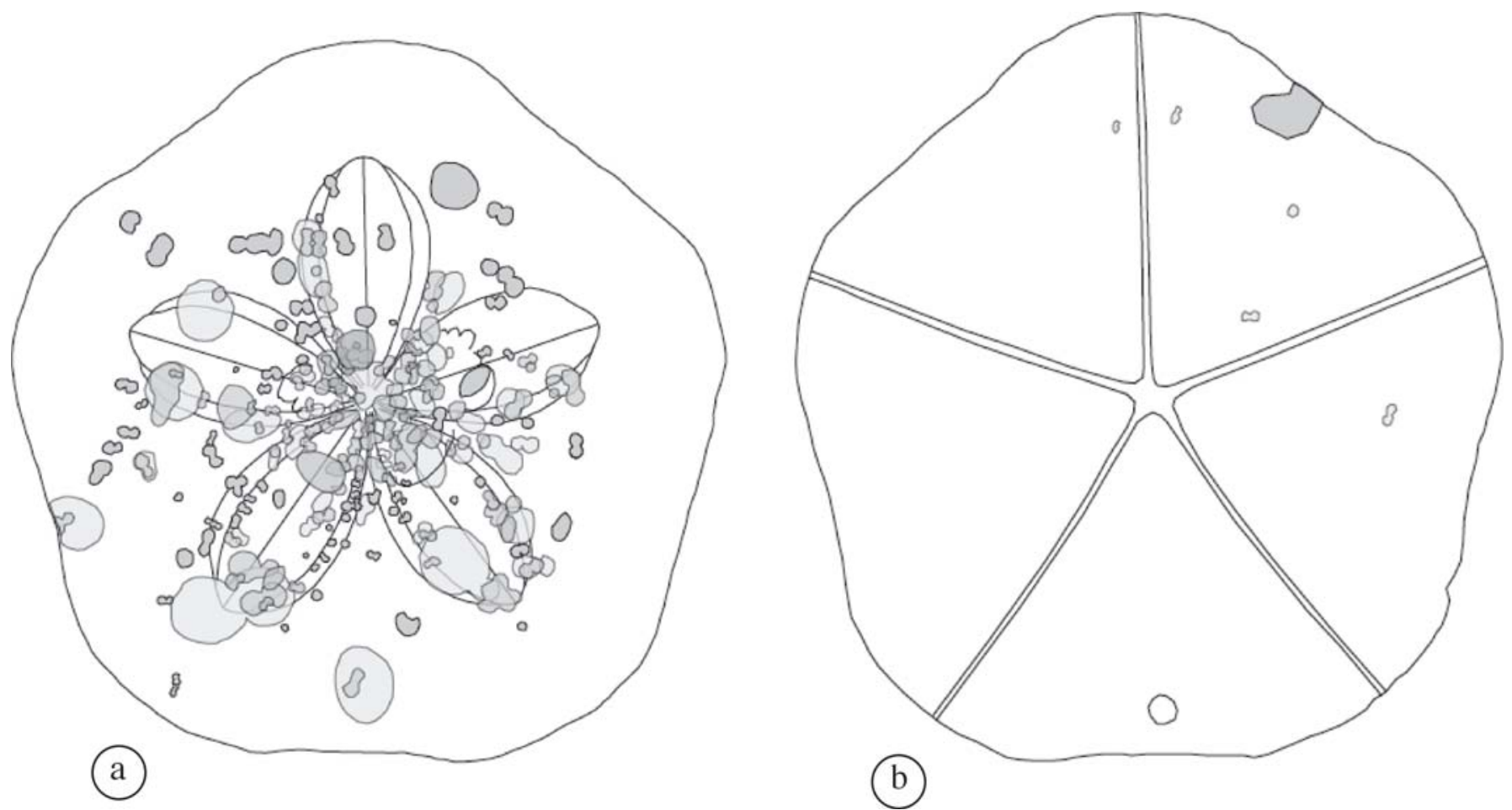

Figura 5. Diagramas de contorno con la distribución de Gastrochaenolites cf. digujus Kelley y Bromley, 1984. a: Superficie aboral. b: Superficie oral.

Sketches showing the distribution of Gastrochaenolites $c f$. digujus Kelley and Bromley, 1984. a: Aboral surface. b: Oral surface.

notables, ya sea en cuanto a la región de la abertura, del cuello, o de la propia forma de la madriguera, de su sección y/u ornamentación interna (Kelley y Bromley, 1984; Bromley y D’Alessandro, 1987; Wilson y Palmer, 1998; Ekdale y Bromley, 2001)

\section{Maeandropolydora sulcans Voigt, 1965}

Figs. 3a; $7 \mathrm{~g}$

Descripción: Perforaciones cilíndricas que muestran dos aberturas, y que discurren irregularmente sobre la superficie del sustrato, con una trayectoria corta y sinuosa. Las perforaciones, a modo de surcos, aparecen desconectadas, sin relación aparente. Los limbos no presentan bolsas o vanos bien definidos. La anchura de los surcos es muy constante a lo largo de su recorrido, con un valor medio de $0,7 \mathrm{~mm}$. La longitud de la pista no excede de $16 \mathrm{~mm}$.

Observaciones: Esta pista es muy escasa y sólo se ha localizado de forma muy dispersa, en la cara aboral de los caparazones (Fig. 7g) de C. cermenatii (Tabla 1), especialmente en los ambulacros. A pesar de su escasa representatividad y corto desarrollo, su diagnosis no admite dudas, ya que presenta las características propias de la icnoespecie cuando existe una disolución de las capas superficiales del sustrato (Bromley y D’Alessandro, 1983).

\section{OTRAS ESTRUCTURAS DE DUROFAGIA}

Al margen de las estructuras anteriores, también se encuentran en ocasiones (un 1,5\% de casos en los ejemplares estudiados) evidencias de depredación muy notables y que consisten en roturas marginales bastante importantes, definidas por profundas incisiones o mordiscos en el ambitus de los caparazones. Estos mordiscos son de escala centimétrica, entre 2,5 y $5 \mathrm{~cm}$ de longitud, y es muy frecuente que puedan reconocerse incluso las señales de los dientes de los depredadores, muy probablemente peces o crustáceos. Aunque pueden aparecer en cualquier zona, son especialmente abundantes en los márgenes de los interambulacros I-III y IV. (Figs. 6, 7h). Los erizos que presentan estos registros sólo son C. cermenatii y Clypeaster sp. (Tabla 1). Es de resaltar que, asociadas a estos traumatismos, aparezcan siempre evidencias de reparación, ya sea aglutinando fragmentos calcáreos para sellar las heridas o bien produciendo secreciones anormales de material secundario que han dado lugar a crecimientos anormales de los caparazones muy espectaculares (Figs. 6, 7h).

\section{CONSIDERACIONESPALEOECOLÓGICAS}

Uno de los aspectos más interesantes que supone el estudio de las estructuras bioerosivas sobre los caparazones 


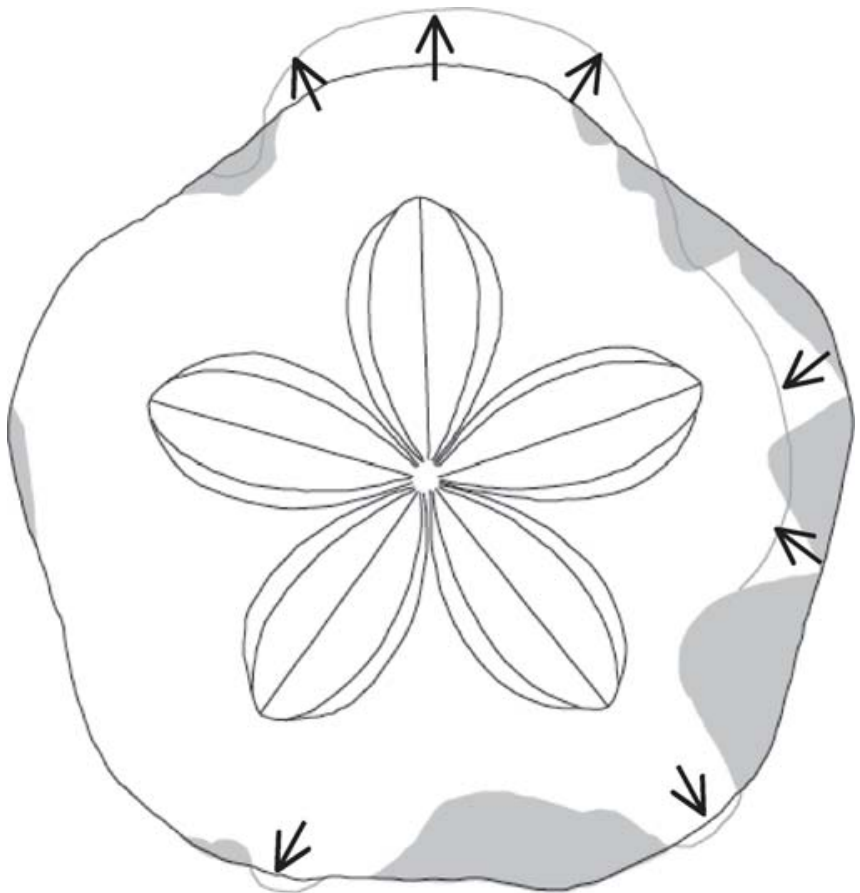

Figura 6. Diagrama de contorno que refleja la posición de los mordiscos (áreas sombreadas) y los crecimientos anormales de material secundario (flechas). Superficie aboral.

Sketch showing the location of the bites (shadow areas) and the secretions of the secondary test material (arrows). Aboral surface.

de los equinoideos es que permiten establecer con bastante fiabilidad no sólo los posibles fenómenos de coacción que se produjeron, sino también la historia post-mortem sufrida por éstos, así como su relación con diversas características del medio, especialmente las que tienen que ver con la energía y las corrientes.

Quizás el caso de coacción mejor registrado, y también el más aparente, es el de la depredación. Este fenómeno es muy frecuente y por tanto, ha sido referenciado detalladamente en la bibliografía específica. (Nebelsick, 1999 a, b; Nebelsick y Kowalewski, 2001).

En los ejemplares estudiados hay dos tipos de estructuras bioerosivas que denotan un comportamiento depredador sobre los erizos clypeasteroideos. Así, la presencia de Oichnus indica casi siempre la actividad depredadora de gasterópodos, tal y como suele ser la explicación más corriente, que no la única, para el productor de este icnogénero. De hecho, en la literatura científica, son muchos los casos que se relacionan con la actividad y el comportamiento de estos organismos. Concretamente, con la actividad depredadora de gasterópodos cassididos, ya sea sobre erizos actuales (McClintock y Marion, 1993; Nebelsick, 1999a; Nebelsick y Kowalewski, 1999, 2001), o fósiles (Woodcock y Kelley, 2001, para el Eoceno o Nebelsick, 1999a y Martínez, 2001, para el Mioceno, entre otros). El hecho de que este tipo de gasterópodos no se haya encontrado en los niveles muestreados, abre la posibilidad de que hayan sido otros los organismos productores de tales perforaciones, quizás pequeños cefalópodos, si bien no hay referencias expresas a éstos en la bibliografía consultada, por lo que presumiblemente su incidencia no sea tan importante.

La otra evidencia clara y directa de ataques son los mordiscos y roturas periféricas en el ambitus de los caparazones. Éstos pueden relacionarse con la acción depredadora de diversos crustáceos como cangrejos, langostas, etc, o bien por otros equinodermos, como las estrellas de mar (Himmelman y Steele, 1971). Otros depredadores activos pueden ser los peces (Gripp, 1929; Kier y Grant, 1965; Sala y Zabala, 1996), o ciertos mamíferos como las nutrias e incluso el hombre (Bowen, 1997; Nebelsick, 1999a). Más información sobre los diferentes tipos de depredadores puede encontrarse en Moore (1956), Quinn (1965), Birkeland y Chia (1971), Zinsmeister (1980), Hughes y Hughes (1971), Tegner y Levin (1983), Courville et al. (1988), McClanahan y Muthiga (1989), Gibson y Watson (1989), Frazer et al. (1991), McNamara (1994), Nebelsick y Kowalewski (1999) y Martínez (2001).

En la mayoría de los ejemplos estudiados, las evidencias corresponden a verdaderos mordiscos, donde se reflejan incluso las dentelladas producidas por los depredadores, muy probablemente peces o crustáceos. Estos ataques casi siempre letales en otro tipo de equinoideos, fueron, sin embargo, superados en todos los casos, ya que los clypeasteroideos tienen un caparazón muy robusto, que además se ve favorecido por el alto poder de regeneración de heridas y traumatismos que son generales en todos los equinodermos (Ebert, 1988). Este hecho ha sido por otra parte, registrado en muchos casos, siendo muy frecuente la reparación de las zonas dañadas tanto en erizos actuales como fósiles (Kier y Grant, 1965; Zinsmeister, 1980; Ali, 1982; Smith et al., 1988). También es muy frecuente que la acción del mordisqueo induzca a una removilización de parte del caparazón, de tal forma que las heridas curen mediante secreciones de material secundario, o adición de fragmentos calcáreos cementados a las mismas. De esta forma, se ocasionan superficies irregulares, e incluso, crecimientos anormales y engrosamientos del caparazón (Abdelhamid, 1999), que pueden localizarse en otras áreas que no son estrictamente las del ataque.

Por otra parte, las depresiones irregulares, áreas deprimidas o distorsionadas y las roturas (especialmente las no marginales) suelen relacionarse con la acción mecánica de impactos en medios muy agitados y turbulentos (Abdelhamid, 1999). En nuestros ejemplares no hay pruebas muy claras, aunque existen algunas evidencias asociadas a Oichnus ichnosp. indet. que quizás pudieran tener un origen similar, si bien no se han observado las típicas grietas o fracturas que suelen rodear o dividir a la posible zona de impacto, así como tampoco se han visto reparaciones claras asociadas a estas fracturas. 

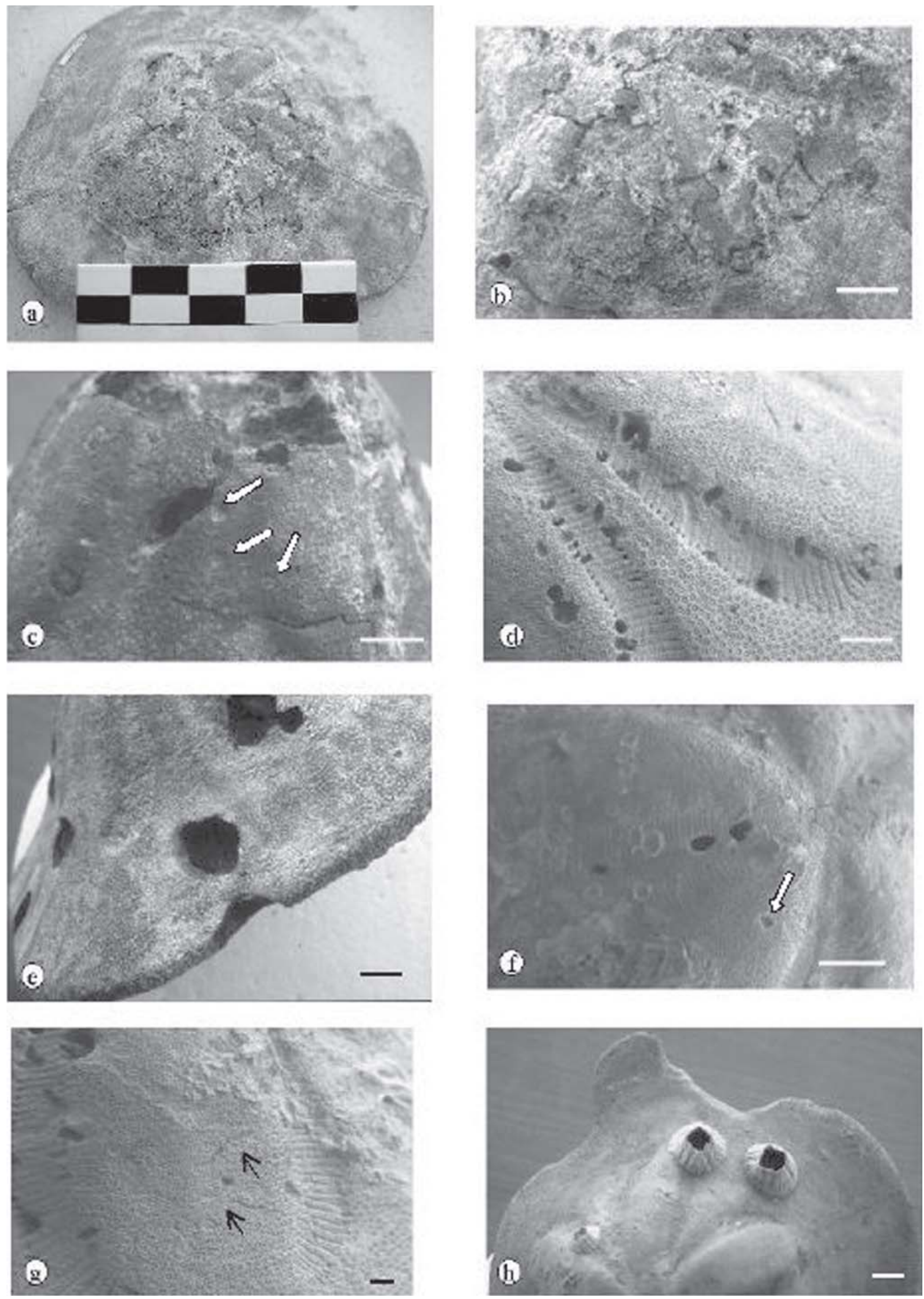
En líneas generales, y a la vista de la distribución de todas las evidencias relacionadas con la actividad depredadora (Figs. 3, 4, 6), se deduce que éstas debieron producirse en dos estados diferentes del sustrato, una cuando los erizos todavía estaban vivos y otra en una fase inmediatamente post-mortem. Al primer caso pertenecerían todas las roturas y mordiscos en el ambitus, puesto que presentan señales inequívocas de haber sido reparados, mientras que en el segundo caso habría que incluir todas las perforaciones relacionadas con Oichnus. Éstos se localizan preferentemente en las zonas poríferas de los ambulacros, lo que indicaría la búsqueda por parte del depredador de las partes blandas más accesibles (los podia). Este comportamiento más que depredador sería necrófago, ya que los erizos no muestran señales de reparación asociadas a las perforaciones (como es habitual en estos casos; Abdelhamid, 1999), ni tan siquiera removilización de material secundario en las áreas adyacentes (Nebelsick, 1999a), por lo que las presas deberían estar ya muertas; eso sí, en una fase muy reciente.

El resto de las estructuras bioerosivas denotan sólo la búsqueda y/o construcción de un domicilio permanente y están representadas por Gastrochaenolites, relacionadas con la actividad perforante de bivalvos litófagos, así como por Maeandropolydora, en relación con la actividad mayoritaria (y presumiblemente) de anélidos. Ambas estructuras se han producido en fases claramente post-mortem del sustrato. En el caso de Gastrochaenolites, éstos se ubican de forma preferente en la zona petaloidea de la cara aboral, que es la región más prominente y elevada del caparazón, una vez que éste queda abandonado al morir el animal. De este modo, los erizos se constituyen en pequeñas "islas" repartidas por el fondo, que son ocupadas masivamente por los bivalvos litófagos y esporádicamente por los anélidos, amén de otro tipo de organismos incrustantes (principalmente balanomorfos). El hecho de que la gran mayoría de las evidencias (el $97 \%$ ) se presente en la cara aboral implica que la energía del medio no debió de ser muy elevada, por lo que los caparazones no llegaron a dar la vuelta (al menos la mayor parte de ellos). Además las evidencias de impactos tampoco son especialmente importantes (1\%), lo cual apoyaría esta hipótesis. Es muy factible que se llegara a conseguir un equilibrio temporal entre la tasa de sedimentación y la energía, de tal forma que a una primera etapa de enterramiento relativamente rápido, que dejara al descubierto la cúpula petaloidea de los caparazones, siguiera una fase de detención o ralentización de la sedimentación, que permitiera la acción depredadora-carroñera de los gasterópodos y la ocupación del sustrato por la mayor parte de los organismos incrustantes (balanomorfos, anélidos) y perforantes (bivalvos litófagos). La fase depredadora no debió de ser muy larga, ya que el porcentaje de ataques es bajo (15\%) frente al grado de ocupación por parte de los bivalvos y anélidos, que es relativamente mucho más significativo (27\%). Estos porcentajes de depredación son anormalmente bajos y se sitúan más en la escala de los valores observados para los erizos del Cretácico que suele ser inferior a un 10\% (Abdelhamid, 1999; Nebelsick y Kowalewski, 2001) o del Paleógeno, entre un 5 y un $10 \%$ (Woodcock y Kelley, 2001). Las razones para esta baja tasa deben estar más en relación con las características propias del medio que con la falta de depredadores potenciales, cuyo número en el Mioceno superior ya era relativamente importante.

Figura 7. a. Vista general de Clypeaster sp. mostrando el área petaloidea del caparazón con Oichnus simplex Bromley, 1981. Ejemplar MGUS-2060. Escala: $10 \mathrm{~cm}$. b. Detalle de la zona petaloidea con Oichnus simplex Bromley, 1981, que han llegado a destruir parcialmente los ambulacros. Escala de la barra: $1 \mathrm{~cm}$. c. Clypeaster portentosus con Gastrochaenolites cf. digujus Kelley y Bromley, 1984 . Las flechas señalan algunos hundimientos del caparazón. Ejemplar MGUS-2061. Escala de la barra: $1 \mathrm{~cm}$. d. Detalle de Clypeaster cermenatii con Gastrochaenolites cf. digujus ubicados en la zona porifera de los ambulacros. Ejemplar MGUS-2070. Escala de la barra:1 cm. e. Detalle de Gastrochaenolites cf. digujus en el borde del ambitus de Clypeaster cermenatii. Ejemplar MGUS-2059. Escala de la barra: $1 \mathrm{~cm}$. f. Gastrochaenolites cf. digujus en el ambulacro de Clypeaster cermenatii. La flecha señala un hundimiento irregular del caparazón. Ejemplar MGUS-2064. Escala de la barra: $1 \mathrm{~cm}$. g. Maeandropolydora sulcans (flechas) en el ambulacro de Clypeaster cermenatii. Ejemplar MGUS-2070. Escala de la barra: $1 \mathrm{~cm}$. h. Mordisco en el ambitus de Clypeaster sp. Ejemplar MGUS-2063. Escala de la barra: $1 \mathrm{~cm}$. a. General view of Clypeaster sp. showing Oichnus simplex Bromley, 1981 on the petalodium zone of the test. Specimen MGUS-2060. Scale bar: $10 \mathrm{~cm}$. b. Detail of the petalodium zone with Oichnus simplex Bromley, 1981 causing partial destruction of the ambulacra. Scale bar: $1 \mathrm{~cm}$. c. Clypeaster portentosus with Gastrochaenolites $c f$. digujus Kelley $y$ Bromley, 1984. Arrows point to some irregular depressions of the test. Specimen MGUS-2061. Scale bar: $1 \mathrm{~cm}$. d. Detail of Clypeaster cermenatii with Gastrochaenolites $c f$. digujus located on the poriferous zone of the ambulacra. Specimen MGUS-2070. Scale bar: $1 \mathrm{~cm}$. e. Detail of Gastrochaenolites $c f$. digujus Kelley y Bromley, 1984 in the ambitus of Clypeaster cermenatii. Specimen MGUS-2059. Scale bar: $1 \mathrm{~cm}$. f. Gastrochaenolites $c f$. digujus on the ambulacrum of Clypeaster cermenatii. Arrow point to an irregular depression of the test. Specimen MGUS-2064. Scale bar: $1 \mathrm{~cm}$. g. Maeandropolydora sulcans (arrows) on the ambulacrum of Clypeaster cermenatii. Specimen MGUS-2070. Scale bar: $1 \mathrm{~cm}$. h. Bite on the ambitus of Clypeaster sp. Specimen MGUS-2063. Scale bar: $1 \mathrm{~cm}$. 


\section{CONCLUSIONES}

Se han analizado y medido un total de 534 estructuras bioerosivas, de las cuales el $97 \%$ se hallan registradas en la cara aboral de los caparazones. El $49 \%$ de los registros reflejan conductas de depredación, el $50 \%$ se refieren a evidencias de búsqueda de domicilio y el $1 \%$ restante se atribuyen a marcas y/o señales irregulares de impactos o de erosión física.

Una de las conductas depredadoras más frecuentes es la relacionada muy probablemente con la actividad de gasterópodos cassiidos, que dio lugar a la producción de Oichnus simplex y Oichnus ichnosp. indet. Otros registros claramente depredadores, y en relación, en su mayoría, con peces o crustáceos, son los mordiscos y/o roturas diversas observadas en los caparazones. Estos últimos ataques se produjeron en vida de los erizos, mientras que los primeros parece ser más probable que se produjeran en fases inmediatamente posteriores a la muerte de éstos (actividad necrófaga), ya que en unos casos hay evidencias de reparación de los traumatismos y en los otros no.

Las conductas que reflejan la construcción de una morada permanente están representadas por los icnotaxones Gastrochaenolites cf. digujus Kelley y Bromley, 1984 y Maeandropolydora sulcans Voigt, 1965. El primero está relacionado con la actividad de bivalvos litófagos y el segundo con la de anélidos poliquetos. Ambos se instalaron en fases claramente post-mortem del sustrato, cuando éstos quedaron semienterrados, con la cúpula aboral de los caparazones sobresaliendo por encima del fondo. La energía del medio no debió de ser muy elevada, ya que las evidencias de impactos y perforaciones en la cara oral de los caparazones son mínimas.

\section{AGRADECIMIENTOS}

Este trabajo ha sido financiado por la Fundação para a Ciência e a Tecnologia en el III Quadro Comunitário de Apoio (Programa PRAXIS XXI) mediante una beca de doctorado (Ref. PRAXIS XXI/BD/21466/99) a Ana Santos, así como por la ayuda al Grupo de Investigación RNM 316 "Tectónica y Paleontología" de la Junta de Andalucía y por el Proyecto BTE2000-0584: "El Neógeno del Mediterráneo Occidental (III): Paleobiología, Paleoclimatología y Bioestratigrafía”. Los autores agradecen también los comentarios y sugerencias aportados por el Dr. Jordi Martinell y D. José Antonio Gámez, que han contribuido a la mejora final del manuscrito.

\section{BIBLIOGRAFÍA}

Abdelhamid, M.A.F. 1999. Parasitism, abnormal growth and predation on Cretaceous echinoids from Egypt. Revue de Paléobiologie de Genève, 18 (1), 69-83.
Ali, M.S.M. 1982. Predation and repairing phenomena in certain clypeasteroid echinoid from the Miocene and Paleocene epochs of Egypt. Journal of the Paleontological Society of India, 27, 7-8.

Bajo, I. 2002. El género Clypeaster (Echinoidea, Clypeasteroidea) en el Mioceno superior de Villanueva del Río y Minas (Sevilla, SO. de España). Revista Española de Paleontología, 17 (2), 177-188.

Birkeland, C. and Chia, F.S. 1971. Recruitment risk, growth, age and predation in two populations of sand dollars, Dendraster excentricus (Eschholtz). Journal of Experimental Marine Biology and Ecology, 6, 265-278.

Bowen, W.D. 1997. Role of marine mammals in aquatic ecosystems. Marine Ecology Progress Series, 158, 267-274.

Bromley, R.G. 1981. Concepts in ichnotaxonomy illustrated by small round holes in shells. Acta Geológica Hispànica, 16, 55-64.

Bromley, R.G., 1993. Predation habits of Octopus. Past and present and a new ichnospecies, Oichnus ovalis. Bulletin of the Geological Society of Denmark, 40, 167-173.

Bromley, R.G. and D'Alessandro, A. 1983. Bioerosion in the Pleistocene of Southern Italy: Ichnogenera Caulostrepsis and Maeandropolydora. Rivista Italiana di Paleontologia i Stratigrafia, 89, 283-309.

Bromley, R. and D'Alessandro, A. 1987. Bioerosion of the Plio-Pleistocene transgresion of Southern Italy. Rivista Italiana di Paleontologia i Stratigrafia, 93, 379-442.

Courville, P., Vincent, E., Thierry, J. et David, B. 1988. La barre à scutelles du Burdigalien (Miocène) de Montbrison-Fontbonau (Bassin de Valréas, Vancluse): du milieu de vie au milieu de dépot. Actes du 6 séminaire international sur les Échinodermes, 3-16.

Ebert, T.A. 1988. Growth, regeneration, and damage repair of spines of the Slate-Pencil sea urchin Heterocentrotus mammilatus (L.) (Echinodermata: Echinoidae). Pacific Science, 42, 3-4.

Ekdale, A.A. and Bromley, R.G. 2001. Bioerosional innovation for living in carbonate hardgrounds in the Early Ordovician of Sweden. Lethaia, 34, 1-12.

Frazer, T.K., Lindberg, W.J. and Stanton, G.R., 1991. Predation on sand dollars by gray Triggerfish, Balistes capriscus, in the northeastern gulf of Mexico. Bulletin of Marine Science, 48, 159-164.

Gibson, M.A. and Watson, J.B. 1989. Predatory and nonpredatory borings in echinoids from the upper Ocala Formation (Eocene), north-central Florida, U.S.A. Palaeogeography, Palaeoclimatology, Palaeoecololy, 71, 309-321.

Gripp, K. 1929. Über Verletzungen an Seeigeln aus der Kreide Norddeutschland. Paläontologische Zeitschrift, 11, 238-245.

Kelly, S.R.A. and Bromley, R.G. 1984. Ichnological nomenclature of clavate borings. Paleontology, 27, 793-807.

Kier, P.M. and Grant, R.E. 1965. Echinoids distribution and habits, Key Largo coral reef preserve. Florida. Smithsonian Miscellaneous Collections, 149, 1-68.

Himmelman, J.H. and Steele, D.H. 1971. Foods and predators of the green sea urchin Strongylocentrotus droebachiensis in Few Foundland waters. Marine Biology, 9, 315-322. 
Hughes, R.N. and Hughes, H. 1971. A study on the gastropod Cassis tuberosa (L.) preying upon sea urchins. Journal of Experimental Marine Biology and Ecology, 7, 305-314.

Lundgren, B. 1891. Studier öfver fossilförande lösa block. Geologiska Föreningen i Stockholm Förhandlinger, 13, 111-121.

Martínez, S. 2001. Predation on the late Miocene Echinoid Monophoraster duboisi (Cotteau, 1884) (Camacho Formation, Uruguay). XVII Congresso Brasileiro de Paleontologia. 5-9 Agosto 2001, Rio Branco, Acre - Universidade Federal do Acre, 16.

McClanahan, T.R. and Muthiga, N.A. 1989. Patterns of predation on a sea urchin, Echinometra mathaei (de Blainville), on Kenyan coral reefs. Journal of Experimental Marine Biology and Ecology, 126, 77-94.

McClintock, J.B. and Marion, K.R. 1993. Predation by the King Helmet (Cassis tuberosa) on Six-Holed Sand Dollars (Leodia sexiesperforata) at San Salvador, Bahamas. Bulletin of Marine Science, 52, 1013-1017.

McNamara, K.J. 1994. The significance of gastropod predation to patterns of evolution and extinction in Australia Tertiary echinoids. In: Echinoderms through Time. (Eds. B. David, A. Guille, J.-P. Féral and M. Roud). Balkema, Rotterdam, 785-793.

Moore, D.R. 1956. Observations on predation on echinoderms by three species of Cassidae. Nautilus, 69, 73-76.

Nebelsick, J.H. 1999a. Taphonomy of Clypeaster fragments: preservation and taphofacies. Lethaia, 32, 241-252.

Nebelsick, J.H. 1999b. Taphonomic legacy of predation on echinoids. In: Echinoderm Research, (Eds. M.D. Candia Carnevalli and F. Bonasoro). Balkema, Rotterdam, 1998, 347-352.

Nebelsick, J.H. and Kowalewski, M. 1999. Drilling predation on Recent Clypeasteroid Echinoids from the Red Sea. Palaios, 14, 127-144.

Nebelsick, J.H. and Kowalewski, M. 2001. Drilling predation on echinoid prey: changes in intensity and site selectivity through time. Abstracts of the North American Paleontological Convention, University of California, Berkeley, 12.

Quinn, B.G. 1965. Predation in sea urchins. Bulletin of Marine Science, 15, 259-264.

Sala, E. and Zabala, M. 1996. Fish predation and the structure of the sea urchin Paracentrotus lividus populations in the NW Mediterranean. Marine Ecology Progress Series, 140, 71-81.

Sierro, F.J., González-Delgado, J.A., Flores, J.A., Dabrio, C.J. and Civis, J. 1990. The Neogene of the Guadalquivir Basin (SW Spain). Paleontologia i Evolució, Memoria Especial, 2, 209-250.

Smith, A.B., Paul, R.C., Gale, A.S. and Donavan, S.K. 1988. Cenomanian and lower Turonian echinoderms from Wilmington, south.east Devon, Englan. Bulletin of the British Museum (Natural History), 42, 245.

Tegner, M.J. and Levin, L.A. 1983. Spiny lobsters and seaurchins. Analysis of a predator-prey interaction. Journal of Experimental Marine Biology and Ecology, 73, 125150.

Voigt, E. 1965. Über parasitische Polychaeten in KreideAustern sowie einige andere in Muschelschalen bohrende Würmer. Paläontologische Zeitschrift, 39, 193-211.

Wilson, M.A. and Palmer, T.J. 1998. The earliest Gastrochaenolites (Early Pennsylvanian, Arkansas, USA): An Upper Paleozoic bivalve boring?. Journal of Paleontology, 72, 769-772.

Woodcock, T.C. and Kelley, P.H. 2001. Predation on irregular echinoids (Eocene) from the Castle Hayne Limestone, Atlantic Coastal Plain (Southeastern North Carolina). Abstracts of the 50 th Annual Meeting, Geological Society of America, Southeastern Section. Raleigh, North Carolina, (U.S.A.), 36.

Zinsmeister, W.J. 1980. Observations on the predation of the clypeasteroid echinoid, Monophoraster darwini, from the upper Miocene Entrerrios Formation, Patagonia, Argentina. Journal of Paleontology, 54, 910-912.

Manuscrito recibido: 11 de diciembre, 2002 Manuscrito aceptado: 25 de febrero, 2003 